\title{
Controlling and Monitoring of Industrial Pressure Process using Distributed Control System
}

\author{
D.Angeline Vijula ${ }^{1}$, V.Thangapandi ${ }^{2}$, K.Vasanth Kumar ${ }^{3}$, B.Vignesh ${ }^{4}$
}

Associate prof., Electronics and Instrumentation Engg.,, Sri Ramakrishna Engineering College, Coimbatore, India ${ }^{1}$

Final year, Electronics and Instrumentation Engineering, Sri Ramakrishna Engineering College, Coimbatore, India ${ }^{2,3,4}$

Abstract: At present time, most of the process industries are in the position to monitor and control many different processes in their manufacturing process at the same instant of time. Global monitoring and controlling of all the process at a same time of instance will lead to increase the process productivity and plant safety. Distributed control and centralized monitoring are the key-factors to ensure the plant safety. This paper aims to enhance the flexibility in controlling and monitoring of industrial pressure process by configuring and developing a HMI using DCS with SCADA. The field output of industrial pressure process are fetched and the parameters have been sent to the Field Control Station (FCS) where the controlling is distributed and monitoring is centralized.Hence the optimized control of different industrial process has been achieved.

Keywords: Distributed Control System (DCS), Human Machine Interface (HMI), Supervisory Control and Data Acquisition (SCADA) and Controller tuning.

\section{INTRODUCTION}

A Distributed Control System is a control system is termed as controlled variable. Manipulated system for a process or plant, wherein control elements are distributed throughout the system. This is in contrast to non-distributed systems, which use a single controller at a central location. In a DCS, a hierarchy of controllers is connected by communications networks for command and monitoring. In Distributed Control System monitoring is centralized and the controlling is distributed. The architecture of DCS is different for different vendors. The architecture of the Siemens DCS is shown Fig.1

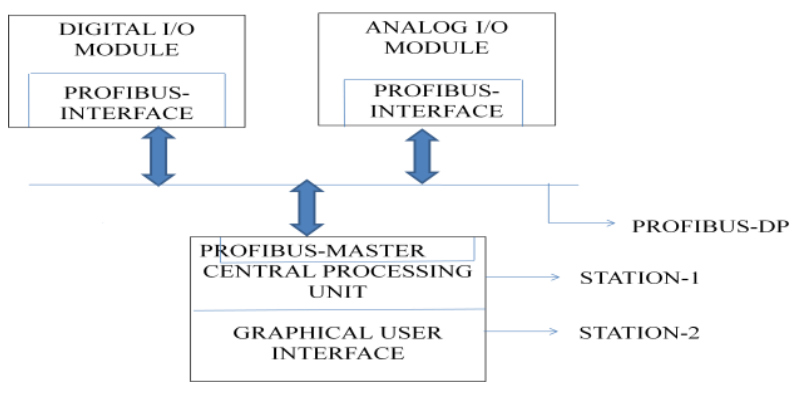

Fig.1. Architecture of SIEMENS DCS

\section{SECTION ORGANISATION}

Section 1 deals with architecture of SIEMENS DCS including various variables of pressure process. Section 2 deals with hardware description. Section 3 deals with SIEMENS DCS. Section 4 deals with controller design. Section 5 deals with discussion on results. Section 6 deals with the conclusion.

\section{PRESSURE PROCESS SECTION}

Pressure is the important physical variable of the industrial process and it is taken in account for control and monitor using SIEMENS DCS.

\section{VARIABLES OF PRESSURE PROCESS STATION}

Controlled variable is pressure inside the chamber. It is the one that must be maintained precisely at the set point. Typically, the variable chosen to represent the state of the variable is inflow rate of the pressure chamber. It is the one that can be changed in order to maintain the controlled variable equal to the set point value. In other words, the variable chosen to control the system's state is termed as manipulated variable. It is also called as controlling variable. Some variables cause disturbances in the process. They are called as load disturbances. The load variable may change either continuously or sporadically with some function of time. Sometimes it is fixed and not a function of time. The load variables are uncontrolled independent variables. When there is a change in these variables, it will upset the control system and their effects can only be corrected in a feedback manner. This means that a change in load variable is not responded by the controller until they upset the controlled variable.

\section{HARDWARE DISCRIPTION}

The hardware setup of the pressure control system is discussed in this chapter

\subsection{BLOCK DIAGRAM}

The pressure of the system is to be maintained at desired value and it is implemented using SIEMENS DCS. The automatic control block of pressure process is shown Fig. 2.

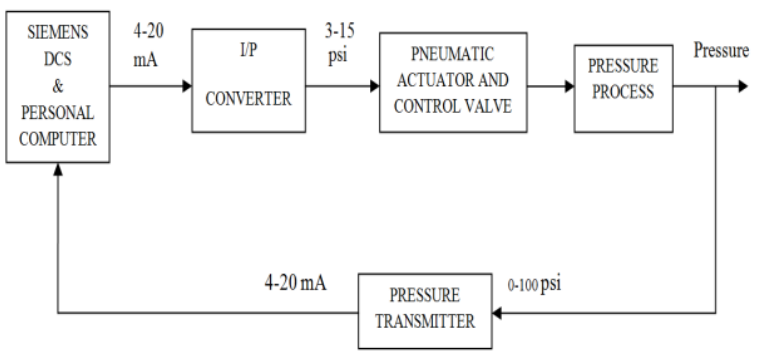

Fig.2. Block diagram of automatic control of pressure process

\subsection{PRESSURE PROCESS}

The pressure process station which is taken for controlling and monitoring is considered here. Pressure 
transmitter senses the process variable (pressure signal) and converts it into 4-20mA current signal. The signal from DAQ will be (4-20) mA current signal. This current signal should be converted into corresponding pressure (315) psi using $\mathrm{I} / \mathrm{P}$ converter. The electronic part module receives standard DC current input signal (4-20) mA from PC. This current will operate coils then increasing or decreasing the magnet force following the input signal. Magnetic force will drive the linkage to adjust the distance between flapper and nozzle or in other word to control bleed air through an integral flapper-nozzle arrangement. The nozzle pressure provides the input signal to operate the relay.

Then relay output pressure is applied directly to the final control element (control valve or actuator auto choke valve). When the flapper moves away from the nozzle, the output pressure decreases and inversely when the flapper come closer to the nozzle the output pressure will increase. The corrective action is applied through the pneumatic actuator by displacement of the control valve. The control valve maintains the level by adjusting the inflow rate. A pneumatic actuator mainly consists of a piston, a cylinder, and valves or ports. The piston is covered by a diaphragm, or seal, which keeps the air in the upper portion of the cylinder, allowing air pressure to force the diaphragm downward, moving the piston underneath, which in turn moves the valve stem, which is linked to the internal parts of the actuator.

Pneumatic actuators may only have one spot for a signal input, top or bottom, depending on action required. This pressure is transferred to the valve stem, which is hooked up to either the valve plug. Larger forces are required in high pressure or high flow pipelines to allow the valve to overcome these forces, and allow it to move the valves moving parts to control the material flowing inside. Valves input pressure is the control signal. This can come from a variety of measuring devices, and each different pressure is a different set point for a valve.

A valve could be controlling the pressure in a vessel which has a constant out flow and a varied in-flow (varied by the actuator and valve). A pressure transmitter will monitor the pressure in the vessel and transmit a signal. As the pressure rises in the vessel, the output of the transmitter rises, this increase in pressure is sent to the valve, which causes the valve to stroke downward, and start closing the valve, decreasing flow into the vessel, reducing the pressure in the vessel as excess pressure is evacuated through the out flow. This is called a direct acting process.

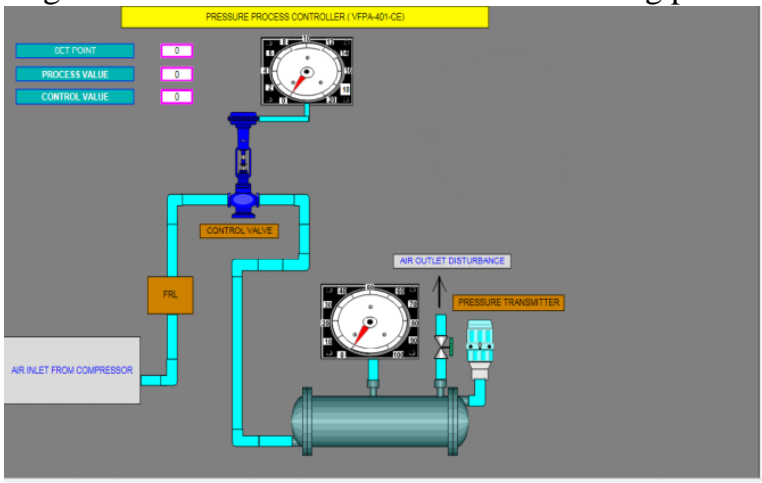

Fig.3. Human Machine Interface for the pressure process

\section{SIEMENS DCS}

The SIEMENS DCS for controlling and monitoring is considered here. The parts of the VDCS-108 3.1ET 200M Distributed I/O Device

SIMATIC ET $200 \mathrm{M}$ is the modular I/O station for the control cabinet with high density- channel applications. Connection to PROFIBUS and PROFINET is achieved using interface modules - optionally for PROFIBUS also using fiber optic cables. The ET 200M can be used for standard as well as failsafe applications. Up to 12 multi-channel signal modules (e.g. 64 digital inputs) and function modules as well as \$7-300 communications processors can be used as I/O modules the interface to the process.ET 200M supports modules with expanded user data, e.g. HART modules with HART minor variables. In addition to proven connection techniques the ET $200 \mathrm{M}$ offers the insulation displacement method Fast Connect for easy wiring.

The ET 200M is particularly suitable for userspecific and complex automation tasks. Applicable Ex analog input or output modules with HART optimize the ET 200M for use in process engineering. Applications that require a higher grade of availability can be realized in combination with redundant systems like S7-400H or S7$400 \mathrm{~F} / \mathrm{FH}$.

\subsection{DIGITAL I/O MODULE}

The Digital I/O modules all have galvanic isolation relative to chassis ground. All modules have LEDs to indicate channel status (ON/OFF) and the standard set of module status indicators. Some output modules and modules with Intrinsic Safety interfaces have indication for the channel error status. The SM 323 (DI/DO-300) modules 24V DC have channels isolated in groups. Each group has a field power status input to indicate presence of field power. Loss of field power is indicated on Warning LED, module status set to warning and channel status set to error. The input module can be configured to monitor field power status. SM 323 Module $24 \mathrm{~V} \mathrm{DC}$ has one isolated group with 16 channels.

\section{Specifications:}

\section{- Make: SIEMENS. \\ Model: SM 323. \\ Input Voltage: $24 \mathrm{~V}$ DC. \\ Digital Inputs: 16 Nos. \\ Digital Outputs: 16 Nos. \\ 3.3 ANALOG I/O MODULE}

Analog I/O SM 334 (AI/AO-300) modules have galvanic isolation relative to chassis ground in a group of 4 channels. Some of the modules also have isolation between channels. The modules have the standard set of module status indicators. Open circuit detection is available for inputs and outputs configured for 0 to $10 \mathrm{~V}$ DC, 0 to $20 \mathrm{~mA}$, and 4 t0 $20 \mathrm{~mA}$. SM 334 Module having 4 Input channels and 2 Output channels.

\section{Specifications:}

$\begin{array}{ll}\text { - } & \text { Make: SIEMENS. } \\ \text { - } & \text { Model: SM } 334 . \\ & \text { Input Voltage: } 24 \text { V DC. }\end{array}$


- $\quad$ Analog Inputs: 4 channels.

- Analog Outputs: 2 channels.

- Voltage Range: 0 to 10 V DC.

- $\quad$ Current Range: 0 to $20 \mathrm{~mA}$ or 4 to $20 \mathrm{~mA}$.

\subsection{PROFIBUS DP COMMUNICATION}

PROFIBUS (Process Field Bus) is a standard for field bus communication in automation technology. PROFIBUS DP runs over two core screened cable that is violet sheathed and its speed varies from $9.6 \mathrm{Kbps}$ to $12 \mathrm{Mbps}$. A particular speed can be chosen for a network to give enough time for communication with all the devices present in the network. If systems change slowly then lower communication speed is suitable and if the systems change quickly then effective communication will happen through faster speed. The RS485 balanced transmission that is used in PROFIBUS DP only allows 32 devices to be connected at once but more devices can be connected and network can be expanded with the use of hubs or repeaters.

The WinLC RTX controller uses a PROFIBUSDP network to communicate with the distributed I/O. The controller is the DP master station, and the I/O modules (for example, ET 200B or ET 200L) are the DP slave stations. An S7 CPU (such as the CPU 315-2 DP) can also function as an intelligent slave device. We use the hardware configuration tools of the programming software to assign the addresses and other parameters for WinLC RTX (DP master) and the I/O (DP slaves).

Like the CP 5512, the CP 5611 A2 is used to connect programming devices and PCs to PROFIBUS up to $12 \mathrm{Mbit} / \mathrm{s}$ and to the multipoint MPI interface of SIMATIC S7: Whereas the CP 5512 is a PC card, the CP 5611 A2 is a PCI card for programming devices/PCs with PCI slot.

\section{CONTROLLER DESIGN}

This chapter deals with the PID controller and Cohen and Coon Tuning method for finding optimum controller parameters.

\subsection{PID CONTROLLER}

A proportional-integral-derivative controller is a control loop feedback mechanism (controller) widely used in industrial control systems. A PID controller calculates an error value as the difference between a measured process variable and a desired set point. The PID controller algorithm involves three separate constant parameters, and is accordingly sometimes called threeterm control: the proportional, the integral and derivative values,

denoted P, I, and D. Simply put, these values can be interpreted in terms of time: P depends on the present error, I on the accumulation of past errors, and $\mathrm{D}$ is a prediction of future errors, based on current rate of change. The weighted sum of these three actions is used to adjust the process via a control element such as the position of a control valve, a damper, or the power supplied to a heating element.

The PID control equation is given by

$$
P=K_{p} e(t)+K_{i} \int_{0}^{t} e(t) d t+K_{d} \frac{d}{d t} e(t)
$$

$\mathrm{K}_{\mathrm{p}}$ - Proportional gain

$\mathrm{P}$ - Controller output

$\mathrm{K}_{\mathrm{i}}$ - Integral gain

$\mathrm{K}_{\mathrm{d}-}$ Derivative gain

$\mathrm{e}(\mathrm{t})$ - error

\subsection{CONTROLLER TUNING}

The objective of the controller tuning is to select proper values for controller parameter to get optimum control. The controller tuning method used in the project is Cohen and Coon controller tuning method.

The tuning is evaluated from open loop response of the process. From the open loop response the parameters such as gain $(\mathrm{K})$, time delay $\left(\mathrm{t}_{\mathrm{d}}\right)$ and time constant $(\tau)$. This method is also called as process reaction curve method or open loop response method.

The controller settings for different control mode are discussed here.

\section{Proportional mode}

Proportional-Integral mode

$$
K_{c}=\frac{\tau}{k t_{d}}
$$

$$
\begin{gathered}
K_{c}=\frac{1}{k} * \frac{\tau}{t_{d}} *\left[0.9+\frac{t_{d}}{12 \tau}\right] \\
T_{i}=t_{d}\left[30+3 \frac{t_{d}}{\tau}\right]
\end{gathered}
$$

Proportional-Integral-Derivative mode

$$
\begin{gathered}
K_{c}=\frac{1}{k} * \frac{\tau}{t_{d}} *\left[\frac{4}{3}+\frac{t_{d}}{4 \tau}\right] \\
T_{i}=t_{d}\left[\frac{\left[32+6 \frac{t_{d}}{\tau}\right]}{13+8 \frac{t_{d}}{\tau}}\right] T_{d}=t_{d}\left[\frac{4}{11+2 \frac{t_{d}}{\tau}}\right]
\end{gathered}
$$

The open loop response values for the pressure process are given below.

\section{$\mathrm{K}=27.04$}

$\mathrm{t}_{\mathrm{d}}=1 \mathrm{sec}$

$\tau=8.8 \mathrm{sec}$

The controller parameters obtained from Cohen and Coon controller tuning method are given below.

$\mathrm{K}_{\mathrm{c}}=0.431$

$\mathrm{K}_{\mathrm{i}}=\frac{1}{T_{i}}=0.34$

$\mathrm{K}_{\mathrm{d}}=\tau_{\mathrm{d}}=0.356$

\section{RESULTS}

The result of controlling pressure process using SIEMENS DCS is discussed.

The PID controller attempts to minimize the error by adjusting the controller output. The PID gain values are calculated by using Cohen and Coon tuning algorithm. The servo operation is also called as set point tracking. The disturbance is considered to be constant for servo 
operation. The closed loop response for set point $=60$ using PID controller of SIMATIC Manager is shown in the Fig.4.

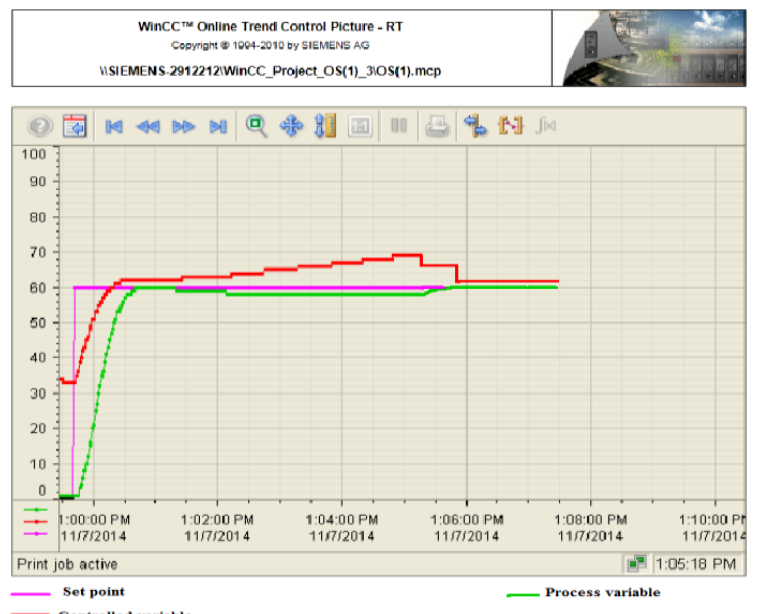

Fig.4. Closed loop response of pressure process by PID controller

Table.1. Performance criteria

\begin{tabular}{|l|c|}
\hline $\begin{array}{l}\text { PERFORMANCECR } \\
\text { ITERIA }\end{array}$ & $\begin{array}{l}\text { PID } \\
\text { CONTROLLER }\end{array}$ \\
\hline Rise time(sec) & 63 \\
\hline Settling time(sec) & 256 \\
\hline Peak time(sec) & 32 \\
\hline
\end{tabular}

\section{CONCLUSION}

Pressure is the real time variable has many impacts on industrial process which is considered here and it is controlled perfectly in PID controller mode by using SIEMENS DCS.DCS gives the industrial platform of controlling and the SCADA provides high level human machine interface .So the perfect control and monitoring of any real time variable is highly achieved using Distributed control system.

\section{REFERENCES}

1. Brendan Galloway, Gerhard Hancke,P. (2008) 'Introduction to Industrial Control Networks',IEEE Transactions on Instrumentation and Measurements Vol.56, No.1, pp.5-10

2. Francesco Adamo, Filippo Attivissiono, Giuseppe Cavone, Nicola Giaquinto (2007), 'SCADA/HMI system in Advance Educational Courses',IEEE Transactions on Instrumentation and Measurements Vol.56,No.1,pp.4-9.

3. George Stephanopoulos,S. (1984) 'Chemical process control ', Prentice - Hall publication , New Jersey , Eastern Economy Edition.

4. GyanRanjanBiswal, Maheshwari R.P., Dewal M.L. (2012)'Modeling, Control and Monitoring of Hydrogen Cooling System in Thermal power plant'IEEE Transactions on Industrial Electronics Vol.59,No.1,pp.562-569.

5. Ivan Castillo, Thomas Edgar,F. (2009) 'Using a DCS for distillation column control in a under graduate unit operation laboratory', American control conference Vol.02, No.5, pp.2744-2745.

6. Kjellsson.J, Vallestad.E.,Steigmann,R. and Dzung,D. (2010), 'Integration of a wireless I/O interface for PROFIBUS and PROFINET for factory automation', IEEE Transactions Vol. 56, No. 10, pp. 4279-4287.

7. Michael L.Lubyen and William Lubyen,L. (1997), 'Essentials of process control', McGraw-Hill publication, Singapore, International Edition.
Osburn ,D.C. (2005) "System and method for communication for a supervisory control and data acquisition (SCADA) system, Vol. 18,No. 4, pp. 1566-157.

9. Thomas, P. Kumar and Chandna, V.K . (2013) 'Design, development, and commissioning of a SCADA laboratory for research and training' IEEE Transactions on Power Systems Vol.19, No.3, pp.1582-1588. 